\title{
Dark nights bring a bright dawn
}

\author{
The relatively dark sky over Africa and the continent's rich human resources are a boon for the growing \\ development initiatives driven by astronomy.
}

$\Lambda$ frica is a continent rich in resources. Take one of the most obvious: land. Africa constitutes roughly $20 \%$ of the world's landmass, and contains $15 \%$ of the world's arable land, yet it only produces $10 \%$ of the world's food. Its resources are underutilized. Improved efficiency can be delivered in a number of ways: merging smaller farmsteads into larger farms, developing transportation infrastructure to make journeys to market more accessible, boosting farming technologies with better seeds, irrigation and fertilizer, and so on. Other repositories of wealth include oil, minerals and most valuably, people - acting both as consumers and as employees in a workforce.

One item that will not be appearing in many lists of African resources is extremely prized by astronomers: access to a dark sky. The night sky generates two precious commodities. The first is inspiration (as suggested by a Comment in this issue by Vanessa McBride et al.). Inspiration is a key driver for education and the power of astronomy to inspire, channelled through projects funded by the International Astronomical Union's Office of Astronomy for Development, can produce observable changes in literacy levels of African children, for instance. Literacy rates in sub-Saharan African adults are some 20 points below the global average. In this way, astronomy can stimulate development in a much-needed area.

Those Africans with a reasonably high level of education have the opportunity to be trained in specialist skills, for instance through the Development in Africa with Radio Astronomy project (see the Comment by Melvin Hoare). This partnership between the UK, South Africa and several other African countries aims to create the first generation of radio astronomers in Africa, including them at the inception of large astronomical operations like the African VLBI Network and the Square Kilometre Array (SKA). The idea behind the project is to nurture self-sustaining professional astronomy groups all over the continent, using, maintaining and developing the upcoming observing facilities. Even those students who choose not to continue in astronomy-related careers will take their experience and technical expertise elsewhere, enriching alternative highly skilled industries.

The other commodity available from a dark sky is data. Data can be mined for knowledge, and knowledge creation is increasingly becoming embraced by developed economies to drive growth. However, to turn electromagnetic radiation from the Universe into data, astronomers need facilities: observatories and telescopes and instruments and computers, and in that sense Africa is also underdeveloped for its size: there are relatively few professional observatories. We learned about Morocco's Oukaïmeden Observatory in our May issue. In this issue, Mirjana Pović et al. give a comprehensive overview of the development of professional astronomy and space science

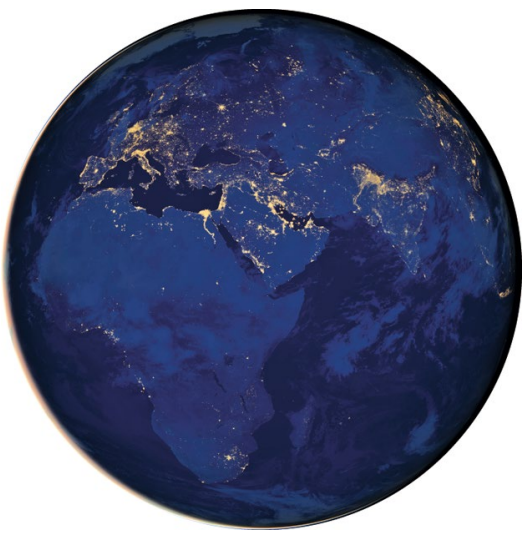

facilities and initiatives in Africa, and the consequential benefits this development will bring in terms of education, reduction of poverty, and attainment of the United Nations Sustainable Development Goals. In addition, we hear in more depth about two southern African telescopes that are converting different kinds of light into scientifically useful information: the High Energy Stereoscopic System (HESS) in Namibia, and the brand-new MeerKAT array in South Africa. HESS is an imaging atmospheric Cherenkov telescope (IACT) array, one of only four IACTs in the world, and the only one in the Southern Hemisphere. Director Mathieu de Naurois provides an overview of the array in a Mission Control. A significantly larger array is MeerKAT, a 64-dish precursor to the midfrequency part of the SKA in neighbouring South Africa. Funded by the South African government, it is a local enterprise that will become a global resource, and is already producing science results (see the Mission Control by Fernando Camilo).

A bright future for African astronomy is connected to interferometry. The Event Horizon Telescope (EHT) Network is an international team striving to image the supermassive black hole at the centre of our Galaxy by linking a number of telescopes across the globe in a very long baseline interferometry (VLBI) experiment. Despite observing runs including the Atacama Large Millimeter/submillimeter Array in the Southern Hemisphere in the last two years, initial indications are that the data will not be sufficient to image the event horizon of Sagittarius A*. This month's cover story examines the ability to test the different theories of gravity using EHT data in its 2017 configuration. A proposal is underway to put a small submillimetre telescope in Namibia - the Africa Millimetre Telescope - that would increase the $u v$ coverage of the EHT by lengthening the east-west extent of the VLBI array in the Southern Hemisphere.

The major initiative in Africa in the next $10-20$ years will be the SKA, which will have dishes in South Africa and eight neighbouring countries, as well as a counterpart in Australia. In South Africa alone, a 12.5 million hectare reserve in the Karoo Desert has been created to host the initial deployment of 133 dishes in a radio-frequency interference-free zone. In the 2030s, this interferometric array will extend to Ghana, Kenya, Zambia, Namibia, Botswana, Madagascar, Mozambique and Mauritius. Many of these countries currently have little or no astronomy activity.

It is clear that Africa has a lot to offer, and with significant investment in intercontinental partnerships and knowledge transfer, Africa's blossoming success in science and technology will benefit itself and the rest of the world.

Published online: 3 July 2018 https://doi.org/10.1038/s41550-018-0535-8 